\title{
Evolution of long-period, white-dwarf binaries: application to GRO J1744-28 and type la supernovae
}

\begin{abstract}
Xiao-Jie $\mathrm{Xu}$ and Xiang-Dong Li
Department of Astronomy, Nanjing University, Nanjing 210093, PR China

e-mail: cruxuxj@gmail.com; lixd@nju.edu.cn

Received 22 May 2008 / Accepted 13 November 2008

\section{ABSTRACT}

We calculated the evolution of long-period white dwarf binaries, by accounting for thermally unstable accretion during mass transfer. Under certain conditions we found that, the accretion rates onto the white dwarf during dwarf nova outbursts can be sufficiently high to allow steady nuclear burning of the accreted matter and growth of the white dwarf mass. If the Chandrasekhar mass is reached, the white dwarf may collapse to be a neutron star, or explode as a type Ia supernova. This scenario appears to account well for the formation of the bursting pulsar GRO J1744-28, and probably type Ia supernovae with delay times considerably longer than 1 Gyr.
\end{abstract}

Key words. accretion, accretion disks - stars: dwarf novae - stars: pulsars: individual: GRO J1744-28 - stars: supernovae: general

\section{Introduction}

Discovered in 1995, GRO J1744-28 is the only X-ray pulsar that exhibitss type-II like X-ray bursts (Fishman et al. 1995; Kouveliotou et al. 1996; Finger et al. 1996). With a spin period of $467 \mathrm{~ms}$, the pulsar is in an 11.8-day binary orbit with a remarkably small mass function $1.3 \times 10^{-4} M_{\odot}$ (Finger et al. 1996), which indicates that, unless we observe the system almost poleon (inclination angle $<15^{\circ}$ ), the companion has a mass $<0.3 M_{\odot}$, implying that GRO J1744-28 is most likely to be a low-mass X-ray binary (LMXB). Using VLT/ISAAC, Gosling et al. (2007) detected two candidate counterparts to GRO J 1744-28, although it was unclear whether one was the true counterpart.

From the observed pulse period and spin-up rate, the magnetic field of GRO J1744-28 can be inferred to be of order $10^{11} \mathrm{G}$ (Finger et al. 1996; Daumerie et al. 1996). Observational evidence for the "propeller" effect in GRO J1744-28 also led to a similar estimate of the pulsar's magnetic field (Cui 1997). Evolutionary calculations by Rappaport \& Joss (1997) implied that the donor star is likely to be a low-mass giant of mass $\sim 0.25 M_{\odot}$. Thus, this system is most likely to be in the final stages of losing its hydrogen-rich envelope, and will become a recycled radio pulsar at the end of the mass transfer.

An important question related to the formation and evolution of GRO J1744-28 is why it has a magnetic field of strength far higher than those of low-mass binary millisecond pulsars, which are generally thought to have evolved from LMXBs. Previous calculations of LMXB evolution indicated that, to form an LMXB in a 12-day orbital period close to the end of the masstransfer stage, the neutron star (NS) is most likely to have accreted at least several tenths of a solar mass (Li \& Wang 1998, and references therein), so that the NS's magnetic field has decayed to $\sim 10^{8}-10^{9} \mathrm{G}$ (Taam \& van den Heuvel 1986; Shibazaki et al. 1989; Urpin \& Geppert 1995; van den Heuvel \& Bitzaraki 1995). To resolve this puzzle, van Paradijs et al. (1997) proposed that the NS could originate from the collapse of a white dwarf (WD) that was accreting from a normal low-mass star, although, according to Li \& Wang (1998) and Sutantyo \& Li (2000), such a NS would still have accreted too much matter after the accretion-induced collapse (AIC) of the WD. The mass transfer rate onto the WD is also far less than that required for steady burning on top of the WD, so that most of the accreting matter would have been expelled from the system during strong $\mathrm{H}$-shell flashes (Kovetz \& Prialnik 1994).

Li \& Wang (1998) proposed a modified scenario for the formation of GRO J1744-28 based on the AIC model. The progenitor system is assumed to be a binary consisting of an $\mathrm{O}-\mathrm{Ne}-\mathrm{Mg}$ WD and $\mathrm{a} \sim 1 M_{\odot}$ main-sequence secondary star in a $\sim 1$ day orbit initially. After several Gyr, the secondary fills its Roche-lobe (RL) due to nuclear expansion. Along with mass transfer, the orbital period increases, and the accretion disk becomes thermally and viscously unstable giving rise to limit cycle behavior (van Paradijs 1996; King et al. 1997; Lasota 2001). As a result, the system appears as a dwarf nova. The mass transfer rate during outbursts could rise to a few times $10^{-8}$ to $10^{-7} M_{\odot} \mathrm{yr}^{-1}$, at which the WD could burn the accreted matter steadily, while in quiescence, little accretion occurs. Thus, the WD could steadily accumulate mass to reach $\sim 1.4 M_{\odot}$ and collapse to become a high-field NS. In the subsequent mass transfer, due to unstable disk accretion the NS must have accreted hardly more than a few hundredths of $1 M_{\odot}$, such that the magnetic field did not decay significantly.

In this work, we attempt to examine the feasibility of the above scenario with detailed numerical calculations of the evolutions of wide WD binaries. However, our interest is not limited to the AIC formation channel to GRO J1744-28 like objects, but extends to possible progenitors of type Ia supernovae (SNe Ia) in old populations. SNe Ia have been used as the most important standard candlelight in cosmology (Philips 1993; Riess et al. 1998; Perlmutter et al. 1999; Riess et al. 2004). It has long been believed that SNe Ia originate from explosions of accreting WDs, whose masses exceed the Chandrasekhar mass limit. However, the nature of the progenitor systems remains unclear. Generally there are two kinds of progenitor-system models: the single degenerate (SD) model in which a WD accretes from a normal binary companion (Nomoto 1982), and the double 
degenerate (DD) model in which two WDs merge due to binary shrinkage (Iben \& Tutukov 1984). The SD model can be divided into two classes: (1) the WD accretes from a main-sequence star more massive than $\sim 2 M_{\odot}$ with initial period $P \sim 1$ day $(\mathrm{Li} \&$ van den Heuvel 1997; Langer et al. 2000; Han \& Podsiadlowski 2004), and (2) the WD accretes from a $\sim 1 M_{\odot}$ red giant star with $P \geq 100$ d (Hachisu et al. 1996; Li \& van den Heuvel 1997). Observations of the circumstellar material in the type Ia SN 2006X inside the Virgo cluster spiral galaxy NGC 4321 strongly suggest that the WD was accreting material from a companion star that was in the red giant phase at the time of the explosion (Patat et al. 2007). It should be emphasized that mass transfer in the evolution of long-period WD binaries is likely to be unstable (at least during part of the mass-transfer lifetime), and this important feature has been ignored practically in previous theoretical work on SNe Ia apart from King et al. (2003), who adopted a similar idea in Li \& Wang (1998) to produce SNe Ia with longperiod dwarf novae in a semi-analytic approach. More detailed work is obviously needed to investigate the influence of the input parameters on the final results.

The structure of this paper is as follows. We describe the binary evolution code and input physics in Sect. 2. In Sects. 3 and 4, we present the calculated results for GRO J1744-28 and the progenitors of SNe Ia, respectively. We discuss the implications of the results and conclude in Sect. 5.

\section{Binary calculations}

\subsection{Binary evolution code}

We used an updated version of the stellar evolution code developed by Eggleton (1971, see also Pols et al. 1995), to calculate the detailed evolution of a binary containing initially a WD of mass $M_{1}$ and a secondary star of mass $M_{2}$. The opacities were taken from Rogers \& Iglesias (1992), and Alexander \& Ferguson (1994) for temperatures below $10^{3.8} \mathrm{~K}$. For the donor star, we assumed a solar chemical composition $(X=0.7, Y=0.28$, and $Z=0.02$ ) and a mixing-length parameter of $\alpha=2.0$. The effective RL radius of the secondary was calculated with Eggleton's equation (Eggleton 1983),

$\frac{R_{\mathrm{L}}}{a}=\frac{0.49 q^{2 / 3}}{0.6 q^{2 / 3}+\ln \left(1+q^{1 / 3}\right)}$,

where $a$ is the orbital separation, and $q=M_{2} / M_{1}$ is the mass ratio. We use the following formula to calculate the mass-transfer rate from the donor star via RL-overflow

$\dot{M}_{2}=-R M T\left(R_{2}-R_{\mathrm{L}}\right)^{3}$,

where $R M T$ is taken to be 500 . We also included stellarwind mass-loss from the donor with the rate suggested by Nieuwenhuijzen \& de Jager (1990),

$$
\begin{aligned}
\log \left(-\dot{M}_{2 \mathrm{w}}\right)= & -14.02+1.24 \log \left(\frac{L_{2}}{L_{\odot}}\right)+0.16 \log \left(\frac{M_{2}}{M_{\odot}}\right) \\
& +0.81 \log \left(\frac{R_{2}}{R_{\odot}}\right),
\end{aligned}
$$

where $L_{2}$ and $R_{2}$ are the luminosity and radius of the donor, respectively.

\subsection{Accretion disk Instability \& accretion rates}

During RL-overflow mass transfer, the accreting material forms a disk surrounding the WD, and the accretion disk may become thermally unstable when the effective temperature in the disk falls below the hydrogen ionization temperature $\sim 6500 \mathrm{~K}$ (van Paradijs 1996; King et al. 1997; Lasota 2001). This also corresponds to a critical mass-transfer rate below which the disk is unstable. As to thermal instability of the accretion disk, we set the critical mass-transfer rate for a stable accretion disk to be

$\dot{M}_{\mathrm{c}, \mathrm{WD}} \simeq 4.3 \times 10^{-9}\left(\frac{P_{\mathrm{orb}}}{4 \mathrm{hr}}\right)^{1.7} M_{\odot} \mathrm{yr}^{-1}$,

for WD accretors, and

$$
\begin{aligned}
\dot{M}_{\mathrm{c}, \mathrm{NS}} \simeq & 3.2 \times 10^{-9}\left(\frac{M_{1}}{1.4 M_{\odot}}\right)^{0.5}\left(\frac{M_{2}}{1 M_{\odot}}\right)^{-0.2} \\
& \times\left(\frac{P_{\text {orb }}}{1 \mathrm{~d}}\right)^{1.4} M_{\odot} \mathrm{yr}^{-1}
\end{aligned}
$$

for NS accretors with an irradiated accretion disk (van Paradijs 1996; Dubus et al. 1999). Here $P_{\text {orb }}$ is the orbital period. If the mass-transfer rate $\left(-\dot{M}_{2}\right)$ is higher than the critical value, we assume that the accretion disk is stable and the compact star accretes smoothly at a rate $\dot{M}_{\mathrm{a}}=-\dot{M}_{2}$; otherwise the compact star accretes only during outbursts and the accretion rate is $\dot{M}_{\mathrm{a}}=-\dot{M}_{2} / d$, where $d$ is the duty cycle, which is typically assumed to be 0.1 to a few $10^{-3}$ (King et al. 2003). We also assume that the accretion rate in both cases is limited to the Eddington accretion rate of the corresponding compact star, and allow the excess mass to be expelled from the binary system, carrying away the specific angular momentum of the compact star.

\subsection{Nuclear burning \& WD mass growth}

After the accumulation of fresh $\mathrm{H}$ and $\mathrm{He}$ on the WD, thermal nuclear burning is likely to occur in certain circumstances. The burning processes of the accreted material on the surface of a WD includes the burning of both $\mathrm{H}$ to $\mathrm{He}$ and $\mathrm{He}$ to heavier elements. To follow the mass growth during the burning processes, we adopted the descriptions in Hachisu et al. (1999), Han \& Podsiadlowski (2004), and Chen \& Li (2007) for H accumulation ratio $\alpha_{\mathrm{H}}$,

$\alpha_{\mathrm{H}}= \begin{cases}\dot{M}_{\mathrm{c}, \mathrm{H}} / \dot{M}_{\mathrm{a}} & \text { if } \dot{M}_{\mathrm{a}}>\dot{M}_{\mathrm{c}, \mathrm{H}} \\ 1 & \text { if } \dot{M}_{\mathrm{c}, \mathrm{H}} \geq \dot{M}_{\mathrm{a}} \geq \dot{M}_{\mathrm{c}, \mathrm{H}} / 8 \\ 0 & \text { if } \dot{M}_{\mathrm{a}}<\dot{M}_{\mathrm{c}, \mathrm{H}} / 8,\end{cases}$

where

$\dot{M}_{\mathrm{c}, \mathrm{H}} \simeq 5.3 \times 10^{-7}\left(\frac{1.7-X}{X}\right)\left(\frac{M_{1}}{1 M_{\odot}}-0.4\right) M_{\odot} \mathrm{yr}^{-1}$.

If $\dot{M}_{\mathrm{a}}<\dot{M}_{\mathrm{c}, \mathrm{H}} / 8$, the transferred matter experiences unstable burning on the surface of the WD and is then expelled in novalike flashes; at higher accretion rates we assume that the mass accumulation rate is limited to $\dot{M}_{\mathrm{c}, \mathrm{H}}$, and for those rates exceeding $\dot{M}_{\mathrm{c}, \mathrm{H}}$, mass is lost from the WD in strong winds. If the WD mass continues to increase in nova-like flashes, almost all WDs in our calculations can reach the Chandrasekhar mass limit sooner or later and become a NS or a SN Ia.

During subsequent He burning, the WD mass also increases only if the He accretion rate $\dot{M}_{\mathrm{He}}=\alpha_{\mathrm{H}} \dot{M}_{\mathrm{a}}$ is within a narrow range. For He burning, we adopted the accumulation ratio $\alpha_{\mathrm{He}}$ in Kato \& Hachisu (2004),

$\alpha_{\mathrm{He}}=\left\{\begin{array}{l}-0.115\left(\log \dot{M}_{\mathrm{He}}+5.7\right)^{2}+1.01 \\ \left(-7.4<\log \dot{M}_{\mathrm{He}}<-6.05\right) \\ 1 \quad\left(-6.05 \leq \log \dot{M}_{\mathrm{He}}\right)\end{array}\right.$ 
for a $1.35 M_{\odot} \mathrm{WD}$,

$\alpha_{\mathrm{He}}=\left\{\begin{array}{l}-0.175\left(\log \dot{M}_{\mathrm{He}}+5.35\right)^{2}+1.03 \\ \left(-7.35<\log \dot{M}_{\mathrm{He}}<-5.83\right), \\ 1 \quad\left(-5.83 \leq \log \dot{M}_{\mathrm{He}}\right),\end{array}\right.$

for a $1.3 M_{\odot} \mathrm{WD}$,

$\alpha_{\mathrm{He}}=\left\{\begin{array}{l}0.54 \log \dot{M}_{\mathrm{He}}+4.16 \\ \left(-7.06<\log \dot{M}_{\mathrm{He}}<-5.95\right), \\ -0.54\left(\log \dot{M}_{\mathrm{He}}+5.6\right)^{2}+1.01 \\ \left(-5.95 \leq \log \dot{M}_{\mathrm{He}}<-5.76\right), \\ 1 \quad\left(-5.76 \leq \log \dot{M}_{\mathrm{He}}\right),\end{array}\right.$

for a $1.1 M_{\odot} \sim 1.2 M_{\odot} \mathrm{WD}$,

$\alpha_{\mathrm{He}}=\left\{\begin{array}{l}-0.35\left(\log \dot{M}_{\mathrm{He}}+5.6\right)^{2}+1.01 \\ \left(-6.92<\log \dot{M}_{\mathrm{He}}<-5.93\right), \\ 1 \quad\left(-5.93 \leq \log \dot{M}_{\mathrm{He}}\right),\end{array}\right.$

for a $1.0 M_{\odot} \mathrm{WD}$,

$\alpha_{\mathrm{He}}=\left\{\begin{array}{l}-0.35\left(\log \dot{M}_{\mathrm{He}}+5.6\right)^{2}+1.07 \\ \left(-6.88<\log \dot{M}_{\mathrm{He}}<-6.05\right), \\ 1 \quad\left(-6.05 \leq \log \dot{M}_{\mathrm{He}}\right),\end{array}\right.$

for a $0.9 M_{\odot} \mathrm{WD}$, and

$\alpha_{\mathrm{He}}=\left\{\begin{array}{l}-0.35\left(\log \dot{M}_{\mathrm{He}}+6.1\right)^{2}+1.02 \\ \left(-6.5<\log \dot{M}_{\mathrm{He}}<-6.34\right), \\ 1 \quad\left(-6.34 \leq \log \dot{M}_{\mathrm{He}}\right),\end{array}\right.$

for a $0.8 M_{\odot}$ WD. The net mass growth rate of the WD is then $\dot{M}_{\mathrm{WD}}=\alpha_{\mathrm{H}} \alpha_{\mathrm{He}} \dot{M}_{\mathrm{a}}$.

\subsection{Mass and angular momentum loss}

To follow the orbital momentum loss, we include the effects of mass loss, magnetic braking (MB), and gravitational wave radiation. The mass loss from the compact star includes those ejected from the system both due to unstable $\mathrm{H}$ and He shell burning and due to super-Eddington accretion. In the WD case, the massloss rate can be expressed as $\dot{M}_{\mathrm{a}}-\alpha_{\mathrm{H}} \alpha_{\mathrm{He}} \min \left(\dot{M}_{\mathrm{a}}, \dot{M}_{\mathrm{E}, \mathrm{WD}}\right)$, where $\dot{M}_{\mathrm{E}, \mathrm{WD}}$ is the Eddington accretion rate of a WD. We allow the ejected matter to carry away the specific orbital angular momentum of the compact star. For MB, we adopt the description of angular momentum loss from Sills et al. (2000) and Andronov et al. (2003),

$\dot{J}_{\mathrm{MB}}= \begin{cases}-K_{\omega} \omega^{3}\left(\frac{R_{2}}{R_{\odot}}\right)^{1 / 2}\left(\frac{M_{2}}{M_{\odot}}\right)^{-1 / 2} & \left(\omega \leq \omega_{\mathrm{cr}}\right), \\ -K_{\omega} \omega \omega_{\mathrm{cr}}^{2}\left(\frac{R_{2}}{R_{\odot}}\right)^{1 / 2}\left(\frac{M_{2}}{M_{\odot}}\right)^{-1 / 2} & \left(\omega>\omega_{\mathrm{cr}}\right),\end{cases}$

where $K_{\omega}=2.7 \times 10^{47} \mathrm{gcms}, \omega$ is the angular frequency of the secondary, and $\omega_{\text {cr }}$ refers to the critical angular frequency above which the MB effect is saturated. Since stars with radiative envelopes do not develop strong magnetic fields, we take account of the MB effect only when the donor is less massive than $1.5 M_{\odot}$. The traditional description of gravitational wave radiation is also included,

$\frac{\mathrm{d}}{\ln } J_{\mathrm{GR}} \mathrm{d} t=-\frac{32}{5} G^{7 / 2} c^{5} \frac{M_{1}^{2} M_{2}^{2}\left(M_{1}+M_{2}\right)^{1 / 2}}{a^{7 / 2}}$,

where $G$ and $c$ are the gravitational constant and speed of light, respectively.

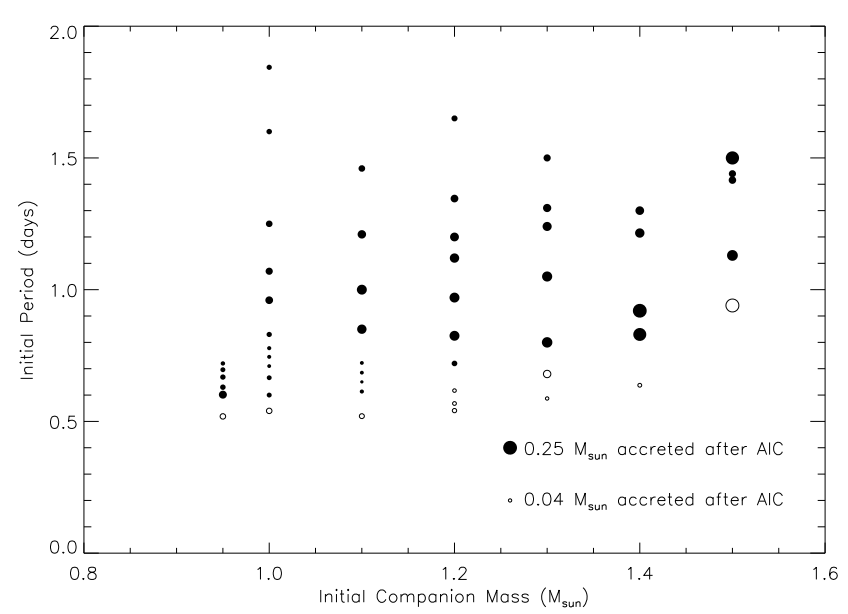

Fig. 1. The initial distribution of the donor masses and orbital periods for binaries evolving with an AIC. The duty cycle of outbursts due to unstable disk accretion is taken to be $d=0.01$. The open and filled dots refer to those with the donors on main sequence and being subgiants at the onset of mass transfer, respectively. The size of the dots indicates the mass that the NS accretes after AIC.

\subsection{Conditions for AIC \& SNe la}

As proposed by Nomoto (1984), an accreting O-Ne-Mg WD of initial mass higher than $1.2 M_{\odot}$ would collapse to a NS if its mass exceeded the Chandrasekhar mass limit, otherwise the WD would end its life as a SN Ia. In our calculations, we define the initial WD mass to be $1.2 M_{\odot}$ for AIC systems, and 0.8-1.0 $M_{\odot}$ for SN Ia systems; the WD is assumed to collapse to become a NS once its mass reaches $1.42 M_{\odot}$, or trigger a SN Ia explosion when its mass reaches $1.38 M_{\odot}$, at which point we also terminate the calculation for SNe Ia systems. For the AIC channel, we assume a $\sim 0.2 M_{\odot}$ mass loss after collapse due to neutrino radiation, so the newborn NS has a mass of $1.22 M_{\odot}$. We also assume the initial magnetic field of the NS to be $B_{\mathrm{i}} \simeq 10^{12} \mathrm{G}$. As a result of the sudden mass loss, the orbital period increases to a higher value, the system becomes detached, and mass transfer pauses. Nuclear evolution will drive the secondary to fill its RL again later, and resumes the mass transfer. The following evolution of the system is similar to that of a normal LMXB. We use Eq. (5) to determine if the accretion disk experiences thermal instability. The evolution of the NS spin and magnetic field caused by mass accretion is calculated according to Li et al. (1998). Specifically, we use a phenomenological form of field decay with accretion, $B \propto B_{\mathrm{i}} /\left(1+\Delta M_{\mathrm{acc}} / m_{\mathrm{B}}\right)$ suggested by Shibazaki et al. (1989), where $\Delta M_{\text {acc }}$ is the mass accreted by the NS, and $m_{\mathrm{B}} \sim 10^{-4} M_{\odot}$.

\section{AIC formation channel to GRO J1744-28}

We first investigate the evolution of a binary containing an $\mathrm{O}-\mathrm{Ne}-\mathrm{Mg}$ WD. The initial WD mass is set to be $1.2 M_{\odot}$ to ensure that the WD collapses to a NS rather than explodes as a SN Ia (Nomoto 1984). The initial mass of the secondary is between $0.9 M_{\odot}$ and $2 M_{\odot}$. We set the duty cycle $d$ to be 0.01 for dwarf nova outbursts. We calculated the evolution of 54 systems with a successful AIC. Figure 1 shows the initial orbital periods and the donor masses of these systems, where the open and filled dots are for systems initiating mass transfer before and after the depletion of central $\mathrm{H}$ of the companion, respectively. The dot size indicates the amount of mass that the NS accreted during its evolution after an AIC. The final distribution 


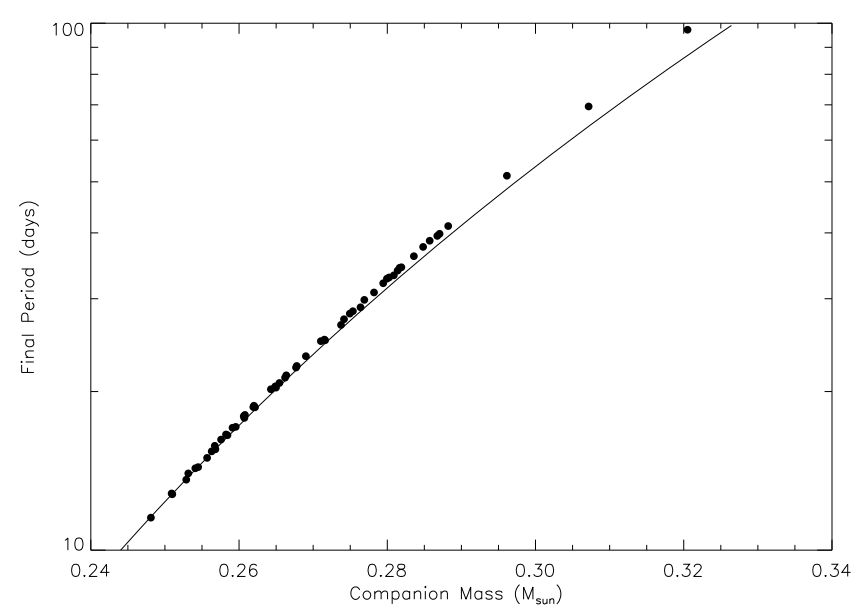

Fig. 2. The end distribution of the companion masses and orbital periods for LMXBs. The filled dots are for LMXBs experienced an AIC from this work, and the solid line indicates the result for normal LMXBs given by Tauris \& Savonije (1999).

of the companion mass $\left(M_{2, \mathrm{f}}\right)$ - orbital period $\left(P_{\text {orb,f }}\right)$ at the end of mass transfer is shown in Fig. 2. To compare with previous works we also plot the $M_{2, \mathrm{f}}-P_{\text {orb,f }}$ relation given by Tauris \& Savonije (1999), which reproduces well our calculated results. Thus from the $M_{2, \mathrm{f}}-P_{\text {orb,f }}$ relation, it appears impossible to determine whether an LMXB system is primordial or originated from the AIC of a WD binary.

Our calculations indicate that the minimum mass a NS accretes after the AIC is $\gtrsim 0.1 M_{\odot}$ when $P_{\text {orb,f }} \sim 12 \mathrm{~d}$, leaving the NS with a weak magnetic field $\left(\sim 10^{8}-10^{9} \mathrm{G}\right)$ (Shibazaki et al. 1989). To solve this problem, we lowered the values of the duty cycle to $d=0.002$ and 0.004 , and found more suitable matches for GRO J1744-28. One possible evolutionary path is given in Fig. 3. The system initially contains a $1.2 M_{\odot} \mathrm{WD}$ and a $1.2 M_{\odot}$ main-sequence companion star with an orbital period of $P_{\text {orb }} \simeq 0.68$ days. Mass transfer initiates at $t \simeq 4.4 \mathrm{Gyr}$ when the companion is about to evolve off the main sequence. The mass transfer rate is always below $10^{-9} M_{\odot} \mathrm{yr}^{-1}$, and the accretion disk is thermally unstable according to Eq. (4). In the first $1.6 \mathrm{Gyr}$, the mass transfer rate is lower than $10^{-9.5} M_{\odot} \mathrm{yr}^{-1}$ and almost all of the accreted mass is lost from the WD due to unstable $\mathrm{H}$ burning. In the next $0.8 \mathrm{Gyr}$, the mass accretion rate is sufficiently high to allow stable $\mathrm{H}$ burning, the WD mass grows to $1.4 M_{\odot}$, and finally collapses to become a NS. During mass transfer the companion loses $\sim 0.7 M_{\odot}$ mass and the orbital period increases to $\sim 2 \mathrm{~d}$. The AIC process further widens the orbit to the one of period $P_{\text {orb }} \simeq 3 \mathrm{~d}$, and the system becomes detached. The companion, now of mass $\sim 0.5 M_{\odot}$, evolves and fills its RL to transfer mass once more at $t \simeq 7$ Gyr. For most of the time, the mass transfer rate is still below $10^{-9} M_{\odot} \mathrm{yr}^{-1}$, so the accretion disk is also thermally unstable, and the binary becomes a soft X-ray transient. Since the mass accretion rate during outbursts is substantially higher than the Eddington limit accretion rate for a NS, most of the accreting matter is expelled from the NS. The orbit expands to $P_{\text {orb }} \simeq 12 \mathrm{~d}$ at $t \simeq 7.4 \mathrm{Gyr}$, when the NS accumulates only $0.013 M_{\odot}$ mass. The system now contains a NS with a relatively strong magnetic field, and a $\sim 0.28 M_{\odot}$ companion. In the future, the mass transfer should continue until the companion loses its entire $\mathrm{H}$ envelope and becomes a WD.

A possible way of testing our model is to search for the counterpart of GRO J1744-28 in other wavelengths, such as optical

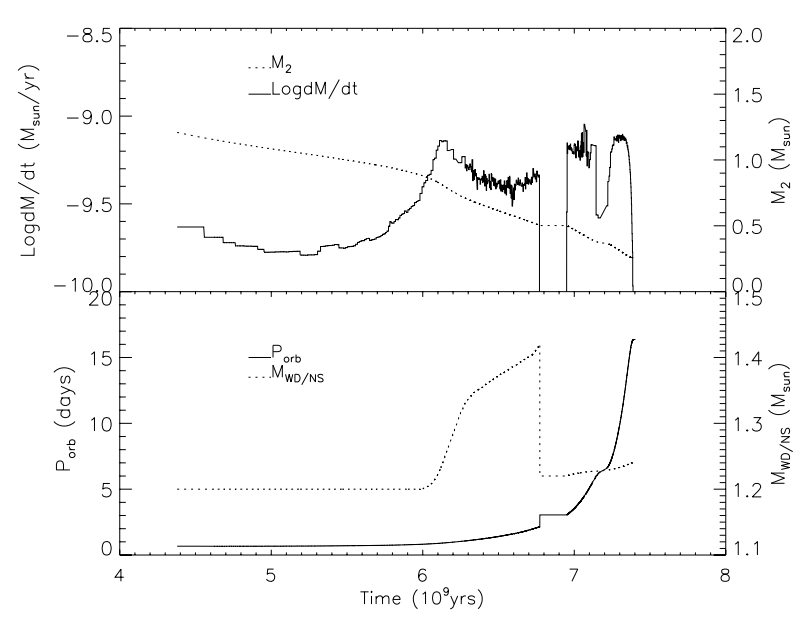

Fig. 3. This figure shows an example of the evolution leading to the formation of GRO J1744-28. The solid and dotted lines show the evolution of the mass transfer rate and the donor mass in the upper panel, and the evolution of the orbital period and the accreting WD/NS mass in the lower panel, respectively.

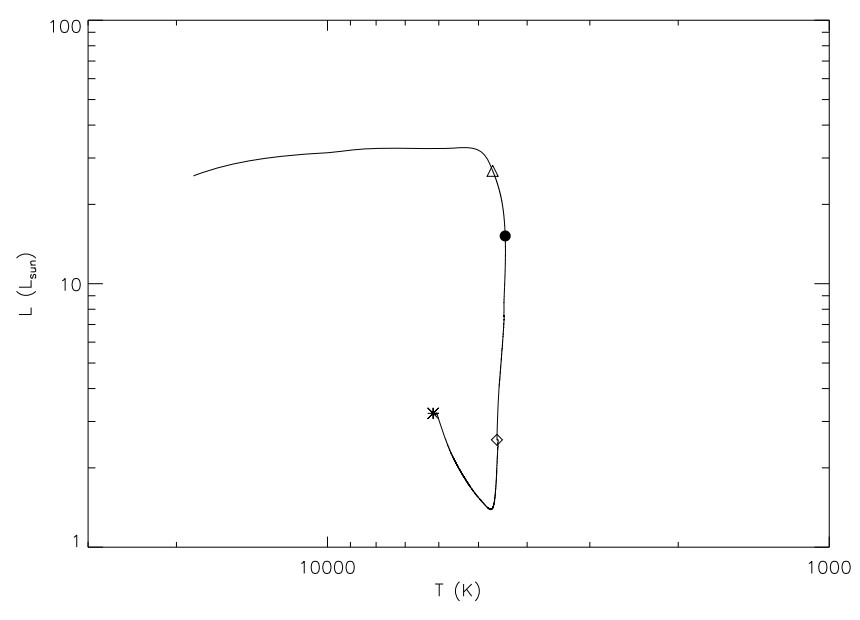

Fig. 4. The evolution of the companion star in H-R diagram. The star, diamond, filled dot and triangle indicate the start of mass transfer, the time of AIC, the present situation and the time when mass transfer terminates, respectively.

or infrared band. We plot the evolutionary path of the companion star in the H-R diagram in Fig. 4, with the star, diamond, filled dot, and triangle symbols indicating the positions of the companion when mass transfer starts, AIC happens, $P_{\text {orb }}=12 \mathrm{~d}$, and mass transfer terminates, respectively. From the figure, the companion star appears to have a surface temperature of $\sim 4400 \mathrm{~K}$ and a luminosity of about $15-30 L_{\odot}$, which is characteristic of a $\mathrm{K}$ giant star. This appears to be consistent with the properties of the bright counterpart detected by Gosling et al. (2007). The fact that its spectrum does not exhibit Brackett- $\gamma$ emission implies that currently the source may not be in an accreting phase, which is also consistent with our expectation of its transient nature.

\section{Formation channel to $\mathrm{SNe}$ la}

To explore possible progenitor systems of SNe Ia, we consider a binary system containing a WD of initial mass $0.8 M_{\odot}$ and 1.0 $M_{\odot}$. The mass of the companion star is taken to range from 


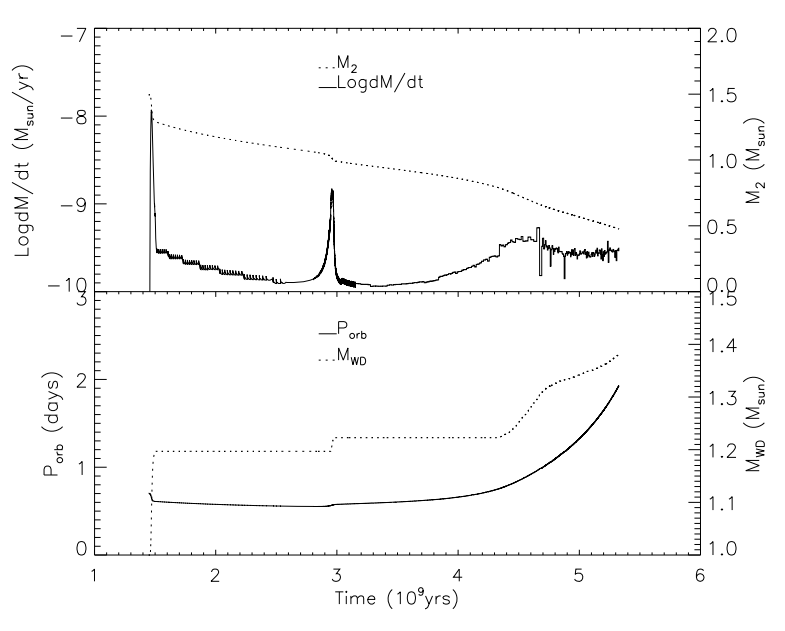

Fig. 5. An example of the evolutionary path to SN Ia. The solid and dotted lines show the evolution of the mass transfer rate and the donor mass in the upper panel, and the evolution of the orbital period and the WD mass in the lower panel, respectively.

$0.9 M_{\odot}$ to $4.0 M_{\odot}$, the initial orbital period from $0.5 \mathrm{~d}$ to $10 \mathrm{~d}$, and the duty cycle is set to be 0.01 for unstable disk accretion.

We terminate the calculation when either of the following conditions is satisfied: (1) the age of the binary exceeds $12 \mathrm{Gyr}$; (2) the mass of the WD reaches $1.38 M_{\odot}$ within $12 \mathrm{Gyr}$ when we assume that it becomes a SN Ia; (3) the mass transfer becomes dynamically unstable. In Fig. 5, we present an example of the evolutionary path to become a SN Ia. The binary consists initially of a WD of mass $1.0 M_{\odot}$ with a $1.5 M_{\odot}$ companion in a $P_{\text {orb }} \simeq 0.7 \mathrm{~d}$ orbit. The companion begins to fill its RL and trigger mass transfer as its core $\mathrm{H}$ burning continues. During the whole evolution, the mass-transfer rate is lower than the critical value given by Eq. (4), and the accretion disk experiences thermal instability. In the first $\sim 10^{8} \mathrm{yr}$, mass transfer occurs on a thermal timescale, since the mass ratio $q>1$ at this stage, which produces a sharp increase in the mass-transfer rate curve and causes the orbit to decrease in physical size. The mass-accretion rate of the WD during outbursts lies above the critical value for stable nuclear burning, and the WD mass increases to $\sim 1.2 M_{\odot}$. After the mass ratio $q$ becomes $<1$, the orbit starts to expand. The mass transfer rate drops below $10^{-9.5} M_{\odot} \mathrm{yr}^{-1}$ in the next $1.4 \mathrm{Gyr}$, and stable $\mathrm{H}$ burning is quenched, such that the mass of the WD stops growing. At $t \simeq 2.9 \mathrm{Gyr}$, the mass transfer rate increases again as the companion evolves off the main sequence and expands, allowing stable nuclear burning, and the mass of the WD increases further to $1.38 M_{\odot}$, triggering a SN Ia at $t \simeq 5.5 \mathrm{Gyr}$. At this time, the companion mass decreases to $\simeq 0.47 M_{\odot}$, and the orbital period becomes $P_{\text {orb }} \simeq 2.0 \mathrm{~d}$.

Figure 6 summarizes the final results of our binary evolution calculations, showing the initial distributions of the SN Ia progenitors in the $M_{2}-P_{\text {orb }}$ diagram. Open dots and triangles in the left and right panel indicate the boundaries of successful systems to produce SNe Ia with WDs of initial mass $1.0 M_{\odot}$ and $0.8 M_{\odot}$, respectively. Systems located inside these regions are able to evolve to become SNe Ia, and those above and below the dotted lines undergo phases of steady accretion and outbursts with steady burning, respectively. For systems located outside the regions, either mass transfer becomes dynamically unstable, leading to a CE phase, or the WD is unable to accrete sufficient matter to trigger a SN Ia explosion within $12 \mathrm{Gyr}$ because the mass transfer rates are too high (i.e., super-Eddington accretion)
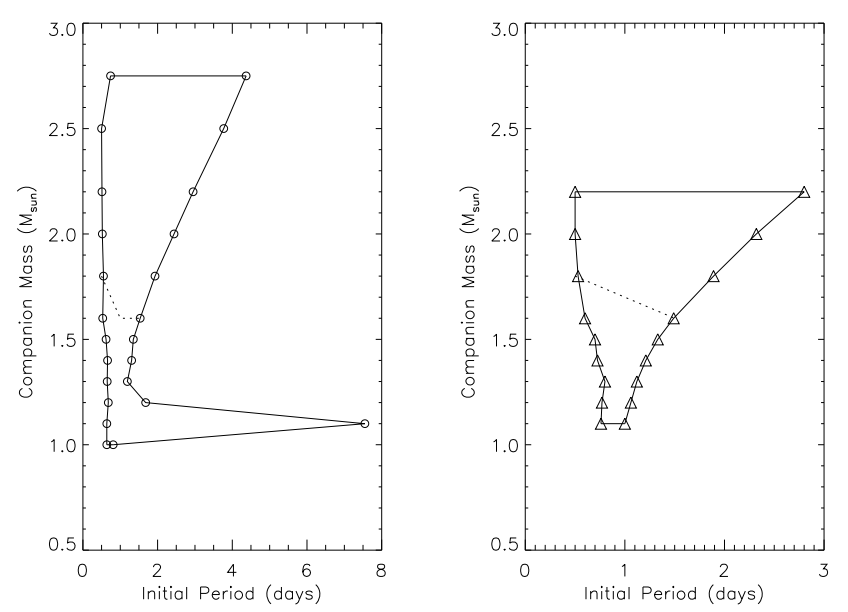

Fig. 6. The initial $M_{2}-P_{\text {orb }}$ distribution for the progenitors of SNe Ia. The open dots and triangles in the left and right panel correspond to systems with $1.0 M_{\odot}$ WDs and $0.8 M_{\odot}$ WDs, respectively. Inside the regions systems above and below the dotted lines undergo steady accretion and outbursts with steady burning, respectively.

or too low during outbursts, so that most of the transferred material is lost from the binaries.

The most striking difference between our results and previous work is that we extend the lower boundary of donor masses to $\sim 1 M_{\odot}$, because we include the effect of a thermally unstable disk on the evolution of WD binaries. Observations of highZ SNe Ia suggest a bimodal delay time distribution, with at least $50 \%$ SNe Ia having delay times longer than $1 \mathrm{Gyr}$ (Mannucci et al. 2006; Dahlen et al. 2008). These tardy SNe Ia are believed to be descendants of systems with donors less massive than $1.5 M_{\odot}$. In our calculations, we find that the delay times are distributed across a rather wide range, from several tenths Gyr to several Gyr. Systems with a $1 M_{\odot}$ WD and relatively massive companion $\left(M_{2} \gtrsim 2 M_{\odot}\right)$ generally have delay times of $\leq 1 \mathrm{Gyr}$, while those with less massive WDs and/or companions have delay times of up to $\sim 6 \mathrm{Gyr}$.

\section{Summary}

Our numerical calculations imply that the properties of the NS in GRO J1744-28 may be accounted for with a scenario involving both AIC of an accreting WD and unstable disk accretion. For long-period dwarf novae, the WD mass may increase during outbursts when the mass accretion rate is sufficiently high to allow stable $\mathrm{H}$ burning, until the Chandrasekhar mass limit is reached. During the subsequent mass transfer after AIC, the newborn NS is also likely to be surrounded by an unstable accretion disk and expels most of the transferred matter, such that its magnetic field does not suffer a significant decay. By including the effect of a thermally unstable accretion disk, we have also explored the possible SNe Ia progenitor systems. The calculated results confirm the suggestion by King et al. (2003) that the disk instability could substantially increase the mass accumulation efficiency for accreting WDs, and cause the possible SNe Ia to occur also in systems with $<1.5 M_{\odot}$ donors.

Of course the results depend on the many input parameters adopted, especially the mass accumulation ratios, the maximum mass-loss rate allowed, and the duty cycles, which are all poorly known. However, these factors influence the binary evolution in different ways. Mass loss can stabilize rapid mass transfer 
when the donor star is a red giant or more massive than the WD (Hachisu et al. 1996; Li \& van den Heuvel 1997), and is closely related to the upper limit to the donor masses. With higher mass accumulation ratios, one may obtain lower mass donor stars in successful systems evolved to AIC or SNe Ia. However, for acceptable values of the mass accumulation ratios, standard binary evolution calculations generally suggest a minimum mass 1.6-2.0 $M_{\odot}$ for the main-sequence donor stars (with solar composition) in SN Ia progenitors (e.g. Li \& van den Heuvel 1997; Langer et al. 2000; Han \& Podsiadlowski 2004). In this respect, thermally unstable disk accretion seems to be a reasonable choice to achieve a donor with mass low as $\sim 1-1.5 M_{\odot}$ in SN Ia progenitors, if a novel mechanism for driving rapid mass transfer in WD binaries with low-mass companions does not exist. In this case, the main uncertainties lie in the fact that the duty cycle varies from one system to another (Lasota 2001), and may evolve with the orbital periods and mass transfer rates. Finally, the duty cycles for accreting NS binaries are likely to differ from those of the WD cases.

Acknowledgements. This work was supported by the Natural Science Foundation of China (under grant number 10873008) and the National Basic Research Program of China (973 Program 2009CB824800).

\section{References}

Alexander, D. R., \& Ferguson, J. W. 1994, ApJ, 437, 879

Andronov, N., Pinsonneault, M., \& Sills, A. 2003, ApJ, 582, 358

Chen, W.-C., \& Li, X.-D. 2007, ApJ, 658, L51

Cui, W. 1997, ApJ, 482, L163

Dahlen, T., Strolger, L.-G., \& Riess, A. G. 2008, ApJ, 681, 462

Daumerie, P., Kalogera, V., Lamb, F. K., \& Psaltis, D. 1996, Nature, 382, 141

Dubus, G., Lasota, J. -P., Hameury, J. M., \& Charles, P. 1999, MNRAS, 303, 139

Eggleton, P. P. 1971, MNRAS, 151, 351

Eggleton, P. P. 1983, ApJ, 268, 368

Finger, M. H., Koh, D. T., Nelson, R. W., et al. 1996, Nature, 381, 291

Fishman, G. J., Kouveliotou, C., van Paradijs, J., et al. 1995, IAU Circ., 6272

Giles, A. B., Swank, J. H., Jahoda, K., Zhang, W., et al. 1996, ApJ, 469, L25
Gosling, A. J., Bandyopadhyay, R. M., Miller-Jones, J. C. A., \& Farrell, S. A. 2007, MNRAS, 380, 1511

Hachisu, I., Kato, M., \& Nomoto, K. 1996, ApJ, 470, L97

Hachisu, I., Kato, M., Nomoto, K., \& Umeda, H. 1999, ApJ, 519, 314

Hameury, J.-M., King, A. R., Lasota, J.-P., \& Raison, F. 1993, A\&A, 277, 81

Han, Z., \& Podsiadlowski, Ph. 2004, MNRAS, 350, 1301

Iben, I. Jr., \& Tutukov, A. V. 1984, ApJS, 54, 335

Kato, M., \& Hachisu, I. 2004, ApJ, 613, L129

King, A. R., Kolb, U., \& Burderi, L. 1996, ApJ, 464, L127

King, A. R., Frank, J., Kolb, U., \& Titter, H. 1997, ApJ, 484, 844

King, A. R., Rolfe, D. J., \& Sshenker, K. 2003, MNRAS, 341, L35

Kouveliotou, C., van Paradijs, J., Fishman, G. J., et al. 1996, Nature, 379, 799

Kovetz, A., \& Prialnik, D. 1994, ApJ, 424, 319

Langer, N., Deutschmann, A., Wellstein, S., \& Höflich, P. 2000, A\&A, 362, 1046

Lasota, J.-P. 2001, NewAR, 45, 449

Li, X.-D., \& van den Heuvel, E. P. J. 1997, A\&A, 322, L9

Li, X.-D., \& Wang, Z.-R. 1998, ApJ, 500, 935

Li, X.-D., van den Heuvel, E. P. J., \& Wang, Z.-R. 1998, ApJ, 497, 865

Mannucci, F., Helfand, A., \& Panagia, N. 2006, MNRAS, 370, 773

Nomoto, K. 1984, in Problems of Colalpse and Numerical Relativity, ed. D. Bancel, \& M. Sifnore (Dordrecht: Reidel), 89

Nomoto, K. 1982b, ApJ, 257, 780

Nieuwenhuijzen, H., \& de Jager, C. 1990, A\&A, 231, 134

Patat, F., Chandra, P., Chevalier, R., et al. 2007, Science, 317, 924

Perlmutter, S., Aldering, G., Goldhaber, G., et al. 1999, ApJ, 517, 565

Philips, M. M. 1993, ApJ, 413, L105

Podsiadlowski, Ph., Rappaport, S., \& Pfahl, E. 2002, ApJ, 565, 1107

Rappaport, S., \& Joss, P. C. 1997, ApJ, 486, 435

Rappaport, S., Podsiadlowski, Ph., Joss, P. C., Di Stefano, R., \& Han, Z. 1995, MNRAS, 273, 731

Rapport, S. A., Vervunt, F., \& Joss, P. C. 1983, ApJ, 275, 713

Riess, A. G., Filippenko, A. V., Challis, P., et al. 1998, AJ, 116, 1009

Riess, A. G., Strolger, L.-G., Tonry, J., et al. 2004, ApJ, 607, 665

Rogers, F. J., \& Iglesias, C. A. 1992, ApJS, 79, 507

Shibazaki, N., Murakami, T., Shaham, J., \& Nomoto, K. 1989, Nature, 342, 656

Sills, A., Pinsonneault, M. H., \& Terndrup, D. M. 2000, ApJ, 534, 335

Sutantyo, W., \& Li, X.-D. 2000, A\&A, 360, 633

Taam, R. E., \& van den Heuvel, E. P. J. 1986, ApJ, 305, 235

Tauris, T. M., \& Savonije, G. J. 1999, A\&A, 350, 928

Urpin, V., \& Geppert, U. 1995, MNRAS, 275, 1117

van den Heuvel, E. P. J., \& Bitzaraki, O. 1995, A\&A, 297, L41

van Paradijs, J. 1996, ApJ, 464, L139

van Paradijs, J., van den Heuvel, E. P. J., Kouveliotou, C., et al. 1997, A\&A, 317, L9 